\title{
ELABORAÇÃO E AVALIAÇÃO DE IOGURTE SABOR FRUTOS DO CERRADO
}

\author{
CLEONICE ROCHA* \\ ROSÁRIO DE MARIAAROUCHE COBUCCI** \\ VALÉRIA RIBEIRO MAITAN*** \\ ORLENE COSTA SILVA****
}

\begin{abstract}
logurtes com adição de doce de araticum, buriti, cagaita, jatobá, mangaba e pequi foram processados, verificando-se sua vida-de-prateleira, preferência e aceitação. Três formulações de iogurtes foram elaboradas, contendo $15 \%, 20 \%$ e $25 \%$ dos doces para os iogurtes de pequi e jatobá e $20 \%, 25 \%$ e $30 \%$ dos doces para os demais iogurtes. $\mathrm{O}$ doce dos frutos foi adicionado ao iogurte natural na temperatura de $\pm 20^{\circ} \mathrm{C}$ e homogeneizado. Após a preparação, as amostras foram resfriadas $a \pm 4^{\circ} \mathrm{C}$ e submetidas à análise de preferência. A amostra preferida foi submetida à análise de aceitação com 100 julgadores não-treinados com idade entre 8 e 65 anos, mediante escala hedônica de nove pontos. Verificou-se a vida-de-prateleira dos iogurtes elaborados durante 7 dias por meio de análise sensorial com equipe composta por 7 julgadores treinados, análise físico-química para verificação do pH e acidez titulável e análise microbiológica para verificação de coliformes totais, coliformes a $45^{\circ} \mathrm{C}$, contagem de estafilococos coagulase positiva e presença de Salmonella sp. em $25 \mathrm{~g}$. Os resultados indicaram boa aceitação dos iogurtes, sendo que o de sabor araticum (com 25\% do doce) apresentou maior média $(7,4)$. Já o iogurte sabor jatobá com $20 \%$ de doce revelou a menor média $(5,49)$. As análises físico-químicas e sensoriais mostraram que os produtos apresentaram-se estáveis sob refrigeração durante 5 a 6 dias, caracterizando-os como produto de consumo rápido. As análises microbiológicas mostraram que as boas práticas de higiene foram adequadas para garantir a qualidade microbiológica dos produtos.
\end{abstract}

PALAVRAS-CHAVE: IOGURTE; ANÁLISE SENSORIAL; VIDA-DE-PRATELEIRA; FRUTOS DO CERRADO.

* Doutora em Química, Professora Titular, Universidade Católica de Goiás (UCG), Goiânia , GO (e-mail: cleonice@ucg.br).

** M. Sc. em Ciência e Tecnologia de Alimentos, Professora Adjunta, UCG, Goiânia, GO (e-mail: rosario@ucg.br).

*** M. Sc. em Biologia, Professora Adjunta, UCG, Goiânia, GO (e-mail: maitan@ucg.br).

**** Doutora em Engenharia Química, Professora Adjunta, Universidade Estadual de Goiás (UEG), Anápolis, GO (e-mail: orlene.costa@hotmail.com). 


\section{INTRODUÇÃO}

O leite de vaca, um dos alimentos naturais mais completos, é rico em nutrientes e contém constituintes de importância nutricional para o homem. O leite e seus derivados formam grupo de grande importância como suprimento nutricional em dietas alimentares (LÓPEZ e COSANO,1995; WONG, LACROIX e ALFORD, 1978).

O iogurte é obtido a partir da fermentação do leite pela ação de cultura láctea mista de Lactobacillus bulgaricus e Streptococcus thermophilus em simbiose, sendo os microorganismos citados viáveis e abundantes no produto final (BRASIL, 2000; FERNANDEZ, et al., 1994). Trata-se de alimento altamente nutritivo, rico em proteínas, $\mathrm{Ca}$, e $\mathrm{P}$, com baixo teor de gorduras e fonte apropriada de minerais como Zn e Mg. É especialmente recomendado para gestantes, lactantes, pessoas idosas ou que necessitem de reposição de cálcio (GARCIA-MARTINEZ et al., 1998; KARAGUL-YUCEER et al., 1999; MARTINEZ et al., 1998; ROJAS et al., 1993). Seu valor nutricional é superior em conteúdo de vitaminas do complexo $\mathrm{B}$ que o leite, sendo mais facilmente aceito por indivíduos com intolerância à lactose (LOURENS-HATTINGH e VILJOEN, 2001).

O leite fermentado apresenta melhor digestibilidade que o leite fresco, pois seus principais constituintes são pré-digeridos devido ao processo fermentativo. Tratando-se de produto de fácil digestão e devido sua riqueza protéica tem sido introduzido no tratamento da inapetência, da alimentação pósoperatória e no caso de transtornos digestivos (GARCIA, RUIZ e DÍAZ, 1986). Também constitui excelente alimento para infantes, período que exige ingestão de minerais. O Ca e P presentes no iogurte são essenciais para boa mineralização dos ossos e dentes (MENOLI et al., 2003) e crianças com deficiência desses minerais podem apresentar raquitismo (PORTO et al., 2005).

Além das propriedades nutricionais, estudos indicam o uso terapêutico do iogurte na prevenção e tratamento de diarréia, redução do colesterol e problemas gastrointestinais (ADOLFSSON, MEYDANI e RUSSEL, 2004; CANZI et al., 2002; GODEL, 2003; JAMES, ANDERSON e GILLILAND, 1999).

A partir de 1996 verificou-se significativo aumento no consumo de produtos lácteos fermentados (MASON, 2006; LÓPEZ et al., 1997; VARNAM e SUTHERLAND, 1995). No Brasil seu consumo ainda é baixo quando comparado com outros países como Chile, França, Irlanda e Bulgária (BRANDÃO, 1995; CONSUMO..., 1997, CASTRO et al., 2006). Entre as causas do baixo consumo pode-se citar o elevado custo do produto, tempo curto de validade e por ser considerado alimento não-essencial (CONSUMO..., 1997; IOGURTES, 1995).

O consumo mundial de iogurte destacou-se na década de 1960 devido à adição de polpa de frutas para atenuação do sabor ácido, resultando em maior aceitação popular do produto (MOREIRA et al., 1999). logurtes com sabores de frutas apresentam maiores teores de minerais (SÁNCHEZ-SEGARRA et al., 2000), que variam conforme o sabor do mesmo (PEDRO, 2001).

O iogurte tradicional com polpa de frutas representa 33\% do mercado brasileiro (CONSUMO..., 1997; IOGURTES..., 1995). Sendo assim, a utilização de frutas do cerrado para saborização de iogurte constitui alternativa alimentar que pode contribuir para maior consumo de frutas e de leite. Maior consumo das frutas do cerrado e sua divulgação também influenciará a preservação da flora da região, rica em espécies úteis ao homem e aos animais silvestres.

Na presente pesquisa teve-se como objetivo elaborar iogurte batido sabor araticum ( Annona crassiflora Mart.), buriti (Mauritia flexuosa Linn. f.), cagaita ( Eugenia dysenterica D.C.), jatobá (Hymenaea stigonocarpa Mart. Ex Hayne), mangaba ( Harconia speciosa Gomes) e pequi (Caryocar brasiliense Camb.), bem como avaliar a vida-de-prateleira e a aceitação dos produtos.

\section{MATERIAL E MÉTODOS}

\subsection{AQUISIÇÃO DOS FRUTOS E DO LEITE}

Os frutos foram adquiridos em feiras livres de Goiânia, ou coletados diretamente da planta em regiões próximas ao município de Goiânia. O leite foi obtido do rebanho bovino, pertencente ao Departamento de Zootecnia da Universidade Católica de Goiás. 


\subsection{PROCESSAMENTO DOS FRUTOS}

O processamento dos frutos constituiu-se, basicamente, nas operações de seleção, higienização, despolpa, cocção e acondicionamento, conforme as boas práticas de fabricação (GUIA..., 2000).

\subsubsection{Obtenção da polpa do frutos}

Os frutos com casca foram higienizados com solução de hipoclorito de sódio a 100 ppm por 10 minutos e descascados manualmente com o uso de facas. Os frutos, com exceção do jatobá, foram submetidos ao branqueamento a $80^{\circ} \mathrm{C}$ por 5 minutos (SILVA, 2000) com o objetivo de inativar as enzimas causadoras de escurecimento e deterioração da matéria-prima. Procedeu-se a retirada da polpa manualmente para araticum, buriti, jatobá e pequi, sendo as polpas de cagaita e mangaba obtidas com o uso de despolpadeira elétrica. As polpas foram acondicionadas em sacos de polietileno, identificadas por rotulagem e armazenadas a $-18^{\circ} \mathrm{C}$. Os utensílios e equipamentos também foram sanitizados por imersão, durante 10 minutos, em solução de hipoclorito de sódio com concentração de 120 ppm.

\subsubsection{Produção dos doces}

Os doces dos frutos foram processados em recipientes de aço inoxidável, utilizando-se fogão semi-industrial (Tabela 1). Após o processamento, os doces foram armazenados em recipientes de vidro com tampas dotadas de garras e conservados em geladeira $\mathrm{a} \pm 4^{\circ} \mathrm{C}$ até utilização.

\section{TABELA 1 - FORMULAÇÃO DOS DOCES DE FRUTOS DO CERRADO}

\begin{tabular}{|c|c|c|c|c|c|c|}
\hline Fruto & $\begin{array}{c}\text { P o Ip a } \\
(\%)\end{array}$ & $\begin{array}{l}\text { A çú car } \\
(\%)\end{array}$ & $\begin{array}{c}\text { Água } \\
\text { Adicionada } \\
(\%)\end{array}$ & $\begin{array}{c}\text { Faixade } \\
\text { Tempo de } \\
\text { Cocção }\end{array}$ & $\begin{array}{c}\text { Concentração } \\
\left({ }^{\circ} \mathrm{B} \text { rix }\right)\end{array}$ & CorlAspecto \\
\hline Araticum & 50,0 & 50,0 & - & $\begin{array}{c}40 \mathrm{~m} \text { in } \\
\mathrm{h}\end{array}$ & $60^{\circ}$ & $\begin{array}{c}\text { alaranjado- } \\
\text { pastoso }\end{array}$ \\
\hline Buriti & 30,0 & 60,0 & 10,0 & $40 \underset{\mathrm{h}}{\mathrm{m}} \mathrm{in}$ a 1 & $60^{\circ}$ & $\begin{array}{l}\text { castanho- } \\
\text { translúcido }\end{array}$ \\
\hline Cagaita & 50,0 & 50,0 & - & $\begin{array}{c}40 \mathrm{~m} \text { in } \\
\mathrm{h}\end{array}$ & $60^{\circ}$ & $\begin{array}{c}\text { esverdeado- } \\
\text { pastoso }\end{array}$ \\
\hline Jatobá & 6,7 & 60,0 & 33,3 & $15 \mathrm{~m}$ in & $60^{\circ}$ & $\begin{array}{l}\text { esverdeado- } \\
\text { translúcido }\end{array}$ \\
\hline Mangaba & 50,0 & 50,0 & - & $40 \underset{\mathrm{h}}{\mathrm{m}}$ in $\mathrm{a} 1$ & $60^{\circ}$ & $\begin{array}{c}\text { am arelado- } \\
\text { pastoso }\end{array}$ \\
\hline Pequi & 23,0 & 62,0 & 15 & $20 \underset{\mathrm{m} \text { in }}{2} \mathrm{a} 1$ & $60^{\circ}$ & $\begin{array}{l}\text { am arelo- } \\
\text { pastoso }\end{array}$ \\
\hline
\end{tabular}

\subsubsection{Processamento do logurte Batido}

Os iogurtes batidos foram processados conforme o fluxograma da Figura 1.

\subsection{ANÁLISES FÍSICO-QUÍMICAS}

As análises físico-químicas foram realizadas com amostras de leite para comprovação de sua qualidade e com amostras dos iogurtes para que fosse determinado o tempo que o produto pode ficar armazenado sem sofrer alterações de suas características químicas. Essas análises consistiram na determinação do $\mathrm{pH}$ por potenciometria direta ( $\mathrm{pHmetro}$ digital com eletrodo de vidro combinado) $\mathrm{e}$ medidas da acidez titulável (titulação potenciométrica com NaOH 0,1 molL-1), sendo os resultados expressos em porcentagem de ácido láctico (IAL, 1985). 


\section{FIGURA 1- FLUXOGRAMA DO PROCESSAMENTO DE IOGURTE BATIDO}

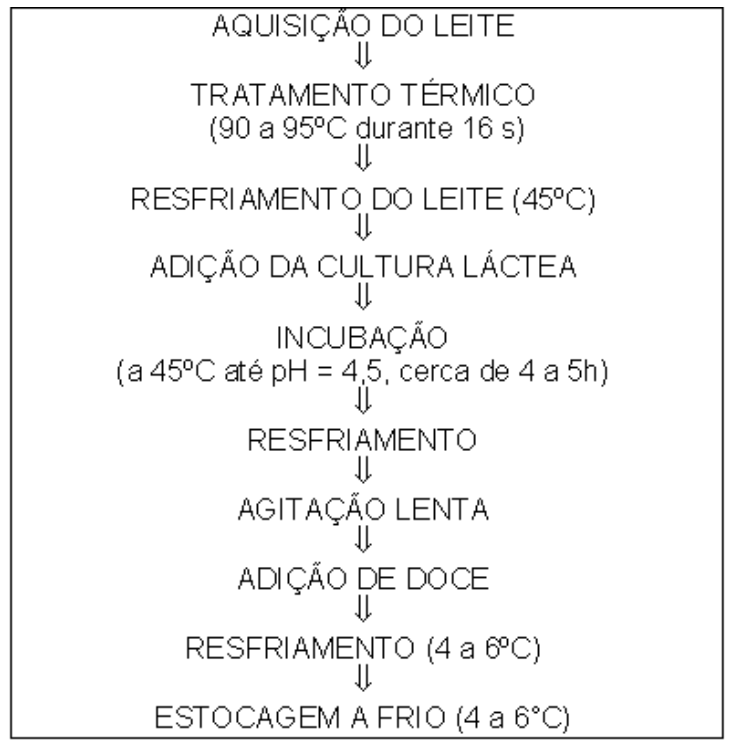

\subsection{ANÁLISES MICROBIOLÓGICAS}

Todas as análises seguiram os métodos preconizados por VANDERZANT e SPLITTSTOESSER (1992) e SILVA, JUNQUEIRA e SILVEIRA (1997). Os resultados obtidos foram confrontados com os padrões microbiológicos estabelecidos pela Agência Nacional de Vigilância Sanitária (BRASIL, 2001). Essas análises consistiram na determinação quantitativa de Salmonella sp, coliformes totais, coliformes a $45^{\circ} \mathrm{C}$ e contagem de estafilococos coagulase positiva.

\subsection{ANÁLISE SENSORIAL}

As análises sensoriais foram realizadas em cabines individualizadas para determinação da vida-de-prateleira, preferência e aceitabilidade dos produtos elaborados.

\subsubsection{Teste de Preferência}

Após testes preliminares, três diferentes formulações foram propostas para cada sabor de iogurte. Em seguida, foram realizados testes de preferência com 90 julgadores não-treinados e faixa etária entre 8 a 65 anos. Três amostras na temperatura de $\pm 4^{\circ} \mathrm{C}$, codificadas com algarismos de três dígitos foram apresentadas simultaneamente aos julgadores para que as ordenassem de acordo com sua preferência. As respostas das ordenações foram registradas em fichas apropriadas (Figura 2), conforme proposta de CHAVES e SPROESSER (1996).

\section{FIGURA 2 - MODELO DE FICHA UTILIZADA NO TESTE DE PREFERÊNCIA}

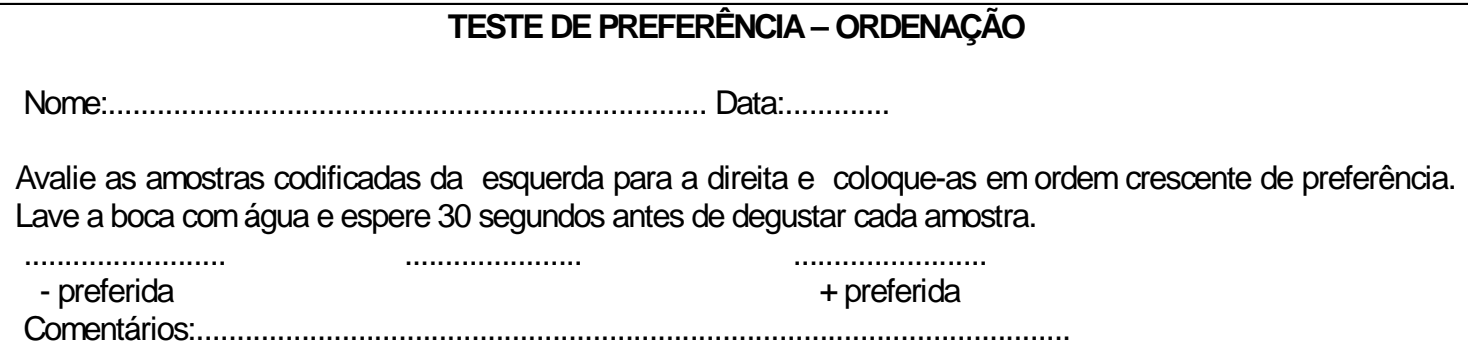


As três amostras foram pontuadas de 1 a 3 , sendo atribuído o valor 1 para a menos preferida e 3 para a mais preferida. Os resultados foram analisados com determinado nível de probabilidade, considerando-se o número de julgamentos e de amostras ordenadas. Os dados das análises de preferência foram tratados utilizando-se a Tabela de NEWELL e MAC FARLANE (1987).

\subsubsection{Testes de Aceitabilidade}

Os testes de aceitabilidade foram realizados com a amostra preferida na temperatura de $\pm 4^{\circ} \mathrm{C}$ e 100 julgadores não-treinados na faixa etária entre 8 e 65 anos. Esses julgaram as amostras, atribuindo notas de 1 a 9 ao produto, conforme a escala FACT (CHAVES e SPROESSER, 1996), apresentada na ficha modelo (Figura 3).

\section{FIGURA 3 - MODELO DE FICHA UTILIZADA NO TESTE DE ACEITABILIDADE}

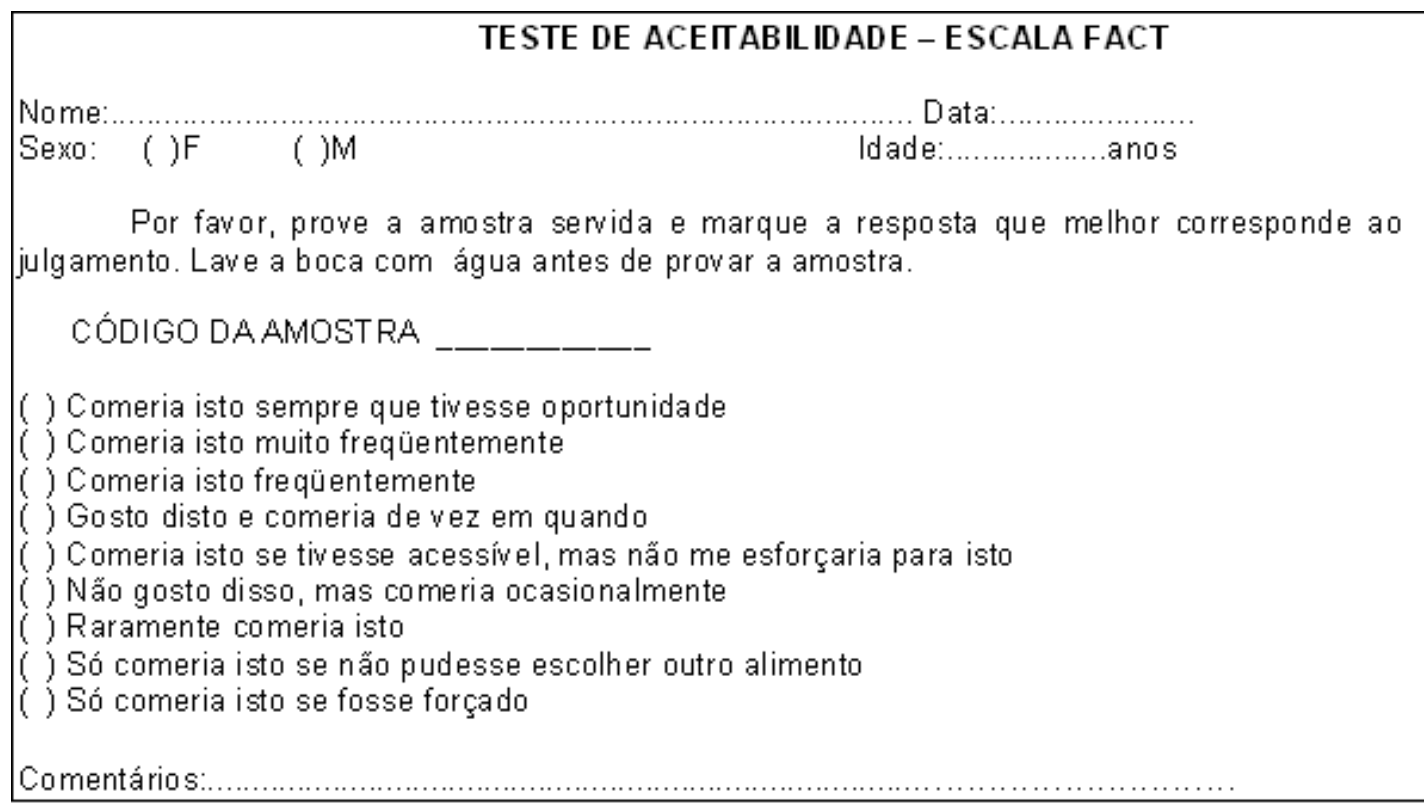

\subsubsection{Análise Sensorial para Determinação da Vida-de-Prateleira}

Para determinar a vida-de-prateleira dos produtos, além das análises físico-químicas, realizouse a análise sensorial durante 7 dias, sendo os produtos mantidos sob refrigeração $\left( \pm 4{ }^{\circ} \mathrm{C}\right)$ em embalagens cobertas por filme plástico.

A metodologia utilizada para avaliação sensorial de vida-de-prateleira dos iogurtes correspondeu ao descrito por FARIA e YOTSUYANAGI (2002) e CHAVES e SPROESSER (1996).

Os testes sensoriais usados para a determinação da estabilidade dos produtos foram realizados com no mínimo seis julgadores treinados. Para o treinamento da equipe foram realizadas reuniões específicas para cada produto. Na primeira sessão foram definidos com a participação da equipe, os atributos a serem estudados e avaliados. Foram também discutidos e explicados os objetivos do treinamento, os procedimentos dos testes e definidas as fichas de avaliação.

Os atributos identificados e avaliados para os iogurtes foram: aparência, odor geral, odor da fruta, consistência na boca, sabor doce, sabor ácido, sabor da fruta e sabor adstringente. Para as análises de odor e sabor utilizou-se escala de 7 pontos com extremos ancorados nos termos (1) muito fraco e (7) muito forte. Para as análises de aparência e consistência utilizou-se escala de 5 pontos (extremos: (1) muito pouco consistente e (5) muito consistente). 
Nas sessões seguintes foram apresentadas as amostras e os materiais de referência para comparação com os extremos das escalas. Para cada atributo foram apresentadas pelo menos duas amostras referências.

A equipe treinada analisou cada um dos iogurtes, durante sete dias consecutivos, registrandose na respectiva ficha a avaliação dos oito atributos.

\subsection{TRATAMENTO DE DADOS}

Os dados das análises físicas, químicas e sensoriais da avaliação da vida-de-prateleira dos produtos elaborados foram tratados estatisticamente por meio de análise de variância (ANOVA) e teste de Tukey ao nível de $5 \%(P<0,05)$. Para o tratamento dos dados das análises físicas e químicas utilizou-se o programa Statistical Analysis System for Windows (SAS,1999) e para os dados da análise sensorial o Sistema para Análise Estatística (SAEG), versão 7.0 (EUCLYDES, 2002).

\section{RESULTADOS E DISCUSSÃO}

\subsection{PROCESSAMENTO DOS IOGURTES}

Os leites utilizados para a elaboração dos iogurtes apresentaram $\mathrm{pH}$ médio 6,5, teor de gordura igual a 3,5 e acidez titulável igual a 1,44 g ácido lático/100 mL, valores enquadrados nos padrões exigidos pela Legislação Brasileira (BRASIL, 2000).

Testes preliminares indicaram as três formulações dos iogurtes a serem usadas, conforme a Tabela 2.

\section{TABELA 2 - FORMULAÇÃO DE IOGURTE BATIDO SABOR FRUTOS DO CERRADO}

\begin{tabular}{c|c|c|c}
\hline Frutos & \multicolumn{3}{|c}{ Doce (\%) } \\
\hline & Form u lação & Form u lação & Form ulaçã o \\
\hline Araticum & $\mathbf{1}$ & $\mathbf{2}$ & $\mathbf{3}$ \\
Buriti & 20 & 25 & 30 \\
Cagaita & 20 & 25 & 30 \\
Jatobá & 20 & 25 & 25 \\
Mangaba & 15 & 25 & 30 \\
Pequi & 20 & 20 & 25 \\
\hline
\end{tabular}

Os iogurtes tiveram tempo de coagulação de 4 horas. O iogurte batido sabor araticum apresentou cor levemente rosada, os sabores cagaita, jatobá e mangaba cor esverdeada, enquanto os iogurtes de pequi e buriti revelaram cor amarela. Todos apresentaram sabor e odor característicos agradáveis.

\subsection{TESTE DE PREFERÊNCIA}

Os dados dos testes de preferência com amostras de iogurte (batido sabores araticum, buriti, cagaita, jatobá, mangaba e pequi) foram tabulados e organizados na forma de gráficos. Os resultados foram analisados a fim de se determinar quais as formulações obtiveram menor e maior preferência na opinião dos 90 julgadores consultados.

Os resultados comparativos das preferências entre as três formulações dos iogurtes processados são apresentados na Figura 4. 


\section{FIGURA 4 - RESULTADOS COMPARATIVOS DO TESTE DE PREFERÊNCIA DOS IOGURTES COM SABOR DE FRUTOS DO CERRADO}

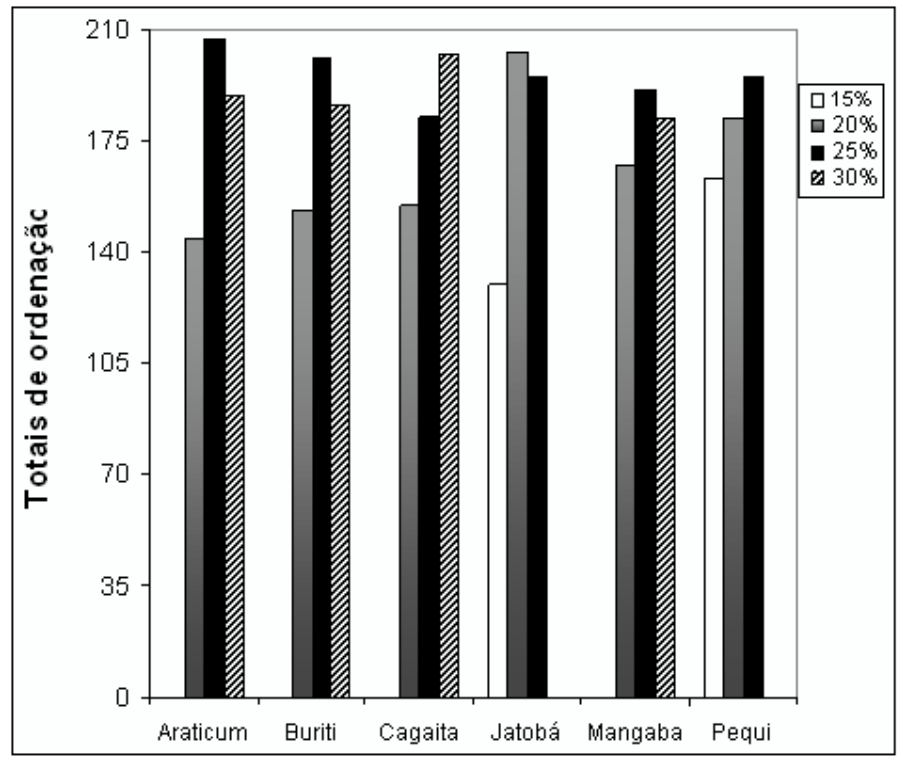

Os resultados dos testes de preferência, submetidos à análise estatística para saber se existiu diferença significativa entre as escolhas de formulação de menor e maior preferência, são apresentados na Tabela 3.

\section{TABELA 3 - RESULTADOS DO TESTE DE PREFERÊNCIA DO IOGURTE BATIDO SABOR BURITI}

\begin{tabular}{l|c|c|c|c}
\hline \multicolumn{5}{c}{ Amostras } \\
\hline \multirow{3}{*}{ Diferença entre os Totais de Ordenação } & & $20 \%$ doce & $25 \%$ doce & $30 \%$ doce \\
\cline { 2 - 5 } & $25 \%$ & $48^{*}$ & - & $15^{\text {ns }}$ \\
\cline { 2 - 5 } & $30 \%$ & $33^{*}$ & $15^{\text {ns }}$ & - \\
\hline
\end{tabular}

ns = não existe diferença significativa entre as amostras ao nível de 5\% de significância.

* = existe diferença significativa entre as amostras ao nível de $5 \%$ de significância ( $\geq 32$, que é a diferença mínima que se deve ter entre os totais de ordenação para que haja diferença significativa ao nível de $5 \%$ de significância de acordo com a Tabela Newell e Mac Farlane (1987), com 3 amostras e 90 julgadores:(3; 90) $=32$.

Para os sabores araticum, buriti, cagaita, mangaba e pequi foram escolhidos para os testes de aceitação as formulações com $25 \%$ de doce por terem sido preferidas e não apresentarem diferença significativa em relação às contendo maior porcentagem de doce. Pelas mesmas razões escolheu-se o iogurte batido sabor jatobá contendo $20 \%$ do doce para os testes de aceitabilidade (Tabela 4 ).

TABELA 4 - FORMULAÇÕES DE IOGURTES COM SABOR DE FRUTOS DO CERRADO UTILIZADOS NOS TESTES DE ACEITABILIDADE

\begin{tabular}{c|c}
\hline Sabor do logurte Batido & Formulação (\% de doce adicionado) \\
\hline Araticum & 25 \\
Buriti & 25 \\
Cagaita & 25 \\
Jatobá & 20 \\
Mangaba & 25 \\
Pequi & 25 \\
\hline
\end{tabular}




\subsection{TESTE DE ACEITABILIDADE}

Os resultados das médias obtidas no teste de aceitabilidade para todos os iogurtes processados podem ser comparados visualizando-se a Figura 5.

\section{FIGURA 5 - MÉDIAS DE ACEITABILIDADE DOS IOGURTES COM SABOR DE FRUTOS DO CERRADO}

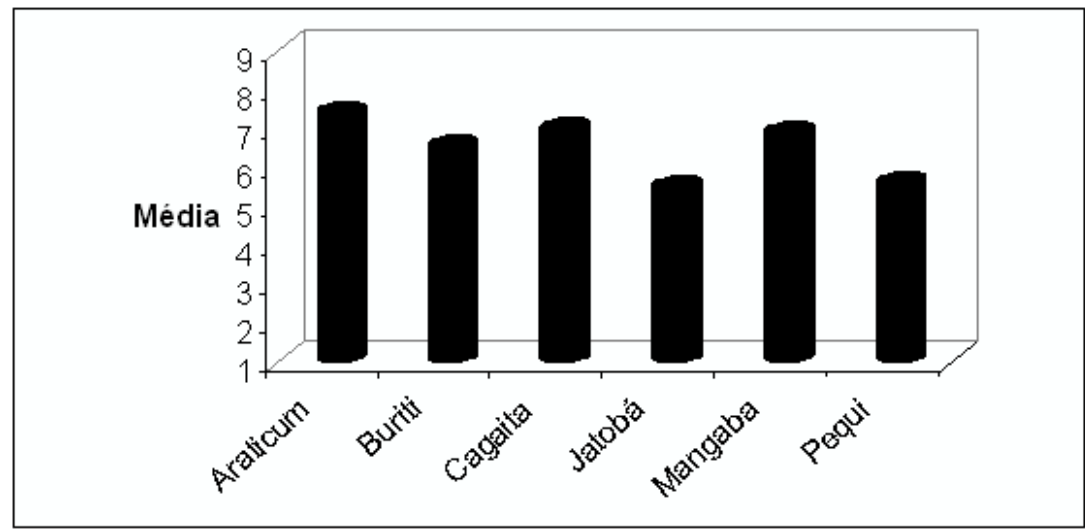

A média obtida na aceitação dos iogurtes (Figura 5) foi comparada com a escala FACT (Figura 3) e os resultados apresentados na Tabela 5.

\section{TABELA 5 - ACEITABILIDADE DOS IOGURTES COM SABOR DE FRUTOS DO CERRADO}

\begin{tabular}{c|c|c}
\hline Sabor do iogurte batido & Média & Associação com a escala FACT \\
\hline Araticum & 7,4 & "comeria isto freqüentemente " \\
Buriti & 6,53 & "gosto disso e comeria de vez em quando" \\
Cagaita & 7,0 & "comeria isto freqüentemente " \\
Jatobá & 5,49 & "comeria isto se tivesse acessivel, mas nâo \\
& & me esforçaria para isto" \\
Mangaba & 6,91 & "comeria isto freqüentemente " \\
Pequi & 5,61 & "gosto disto e comeria de vez em quando" \\
\hline
\end{tabular}

Os iogurtes com sabor jatobá e pequi foram os menos aceitos pelos julgadores, apesar de não terem sido totalmente rejeitados. Os demais produtos apresentaram boa aceitação, estando de acordo com os resultados da aceitabilidade de iogurte de leite de búfala com sabor araticum, mangaba e cagaita (ROCHA et al., 2004)

\subsection{ANÁLISE DE VIDA-DE-PRATELEIRA}

\subsubsection{Análises físico-químicas}

As variações do $\mathrm{pH}$ e acidez titulável dos iogurtes com sabor de frutos do Cerrado durante os sete dias de análises são apresentadas nas Tabelas 6 e 7.

\subsubsection{Análise de acidez titulável}

Os resultados das análises de acidez titulável dos iogurtes, durante os sete dias de armazenamento, são apresentados na Tabela 6. 


\section{TABELA 6 - VARIAÇÃO DE ACIDEZ TITULÁVEL DOS IOGURTES COM}

SABOR DE FRUTOS DO CERRADO

\begin{tabular}{c|cccccc}
\hline \multicolumn{7}{c}{ ACIDEZ (g ácido lácticol100 mL) } \\
\hline $\begin{array}{c}\text { Tempo } \\
\text { (dias) }\end{array}$ & $\begin{array}{c}\text { Iogurte } \\
\text { sabor } \\
\text { araticum }\end{array}$ & $\begin{array}{c}\text { Iogurte } \\
\text { sabor } \\
\text { buriti }\end{array}$ & $\begin{array}{c}\text { logurte } \\
\text { sabor } \\
\text { cagaita }\end{array}$ & $\begin{array}{c}\text { Iogurte } \\
\text { sabor } \\
\text { jatobá }\end{array}$ & $\begin{array}{c}\text { logurte } \\
\text { sabor } \\
\text { mangaba }\end{array}$ & $\begin{array}{c}\text { logurte } \\
\text { sabor } \\
\text { pequi }\end{array}$ \\
\hline 1 & 0,63 & 0,66 & 0,66 & 0,59 & 0,80 & 0,58 \\
2 & 0,66 & 0,72 & 0,69 & 0,71 & 0,82 & 0,57 \\
3 & 0,68 & 0,73 & 0,69 & 0,66 & 0,83 & 0,65 \\
4 & 0,67 & 0,66 & 0,69 & 0,66 & 0,83 & 0,73 \\
5 & 0,68 & 0,69 & 0,68 & 0,70 & 0,82 & 0,78 \\
6 & 0,68 & 0,72 & 0,67 & 0,72 & 0,82 & 0,83 \\
7 & 0,68 & 0,73 & 0,68 & 0,74 & 0,83 & 0,85 \\
\hline
\end{tabular}

Os resultados das análises de $\mathrm{pH}$ obtidos para as amostras dos iogurtes processados são apresentados na Tabela 7.

TABELA 7 - VARIAÇÃO DE pH DOS IOGURTES COM SABOR DE FRUTOS DO CERRADO

\begin{tabular}{|c|c|c|c|c|c|c|}
\hline & \multicolumn{6}{|c|}{$\mathrm{pH}$} \\
\hline $\begin{array}{l}\text { Tempo } \\
\text { (dias) }\end{array}$ & $\begin{array}{l}\text { logurte } \\
\text { sabor } \\
\text { araticum }\end{array}$ & $\begin{array}{c}\text { logurte } \\
\text { sabor } \\
\text { buriti }\end{array}$ & $\begin{array}{l}\text { logurte } \\
\text { sabor } \\
\text { cagaita }\end{array}$ & $\begin{array}{l}\text { logurte } \\
\text { sabor } \\
\text { jatobá }\end{array}$ & $\begin{array}{l}\text { logurte } \\
\text { sabor } \\
\text { mangaba }\end{array}$ & $\begin{array}{l}\text { logurte } \\
\text { sabor } \\
\text { pequi }\end{array}$ \\
\hline 1 & 4,37 & 4,26 & 4,23 & 4,28 & 4,19 & 4,32 \\
\hline 2 & 4,36 & 4,14 & 4,23 & 4,31 & 4,16 & 4,38 \\
\hline 3 & 4,35 & 4,07 & 4,23 & 4,27 & 4,15 & 4,32 \\
\hline 4 & 4,32 & 4,03 & 4,19 & 4,26 & 4,15 & 4,26 \\
\hline 5 & 4,30 & 4,07 & 4,18 & 4,21 & 4,14 & 4,34 \\
\hline 6 & 4,31 & 4,05 & 4,18 & 4,22 & 4,14 & 4,29 \\
\hline 7 & 4,30 & 4,05 & 4,18 & 4,19 & 4,13 & 4,25 \\
\hline
\end{tabular}

Todas as amostras mostraram ligeiro aumento da acidez titulável e diminuição do pH, provavelmente devido ao desenvolvimento das bactérias lácticas da cultura adicionada, que mesmo em baixas temperaturas não têm seu crescimento totalmente inibido (LOURENS-HATTINGH e VILJOEN, 2001). As variações de $\mathrm{pH}$ não foram significativas no nível de $5 \%$ de confiança conforme previsto para iogurtes com adição de sabor (ORTIZ, 1982), entretanto, as variações de acidez titulável mostraramse significativas ao nível de $5 \%$ de confiança. Apesar disso, os valores de $\mathrm{pH}$ e acidez titulável enquadram-se na faixa estabelecida pela legislação (BRASIL, 2000).

\subsubsection{Análises microbiológicas}

Os iogurtes com sabor de frutos do Cerrado submetidos às análises microbiológicas apresentaram, durante a avaliação da vida-de-prateleira, os resultados mostrados na Tabela 8.

Os iogurtes submetidos às análises microbiológicas, durante a vida-de-prateleira, estão de acordo com os padrões microbiológicos legais vigentes quanto ao NMP de coliformes a $45^{\circ} \mathrm{C}$ e Salmonella sp. (BRASIL, 2001). Não há padrões para estafilococos coagulase positivas e coliformes totais na referida resolução, porém a análise de estafilococos indica que as condições higiênicas de produção do alimento foram satisfatórias. Esse resultado refere-se à análise de uma amostra indicativa $(n=1)$. 
TABELA 8 - AVALIAÇÃO MICROBIOLÓGICA DE AMOSTRAS DE IOGURTE BATIDO

\begin{tabular}{|c|c|c|c|c|}
\hline logurte & $\begin{array}{c}\text { NMP } \\
\text { Coliform es } \\
\text { totais } / g\end{array}$ & $\begin{array}{c}\text { N M P } \\
\text { coliform es a } \\
45^{\circ} \mathrm{C} / \mathrm{g}\end{array}$ & $\begin{array}{c}\text { Contagem de } \\
\text { estafilococos } \\
\text { coagulase } \\
\text { positivalg }\end{array}$ & $\begin{array}{c}\text { Sa Im o n e IIa } \\
\text { sp } / 25 \mathrm{~g}\end{array}$ \\
\hline A raticum & $2,1 \times 10^{2}$ & $<3,0$ & A usente & negativo \\
\hline Buriti & $<0,3$ & $<0,3$ & A usente & n e g a tivo \\
\hline $\mathrm{M} a \mathrm{ag} \mathrm{ab}$ & $1,2 \times 10^{2}$ & $<3,0$ & A usente & negativo \\
\hline$P$ e qu i & $1,2 \times 10^{2}$ & $<3,0$ & A usente & negativo \\
\hline
\end{tabular}

\subsubsection{Análises sensoriais}

O iogurte sabor araticum não apresentou variação significativa ao nível de 5\% para os atributos odor (geral e da fruta), sabor doce e consistência na boca. Entretanto, observou-se aumento de acidez

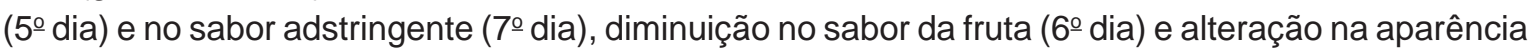
a partir do $2^{\circ}$ dia.

Para o iogurte sabor jatobá observou-se diferença significativa nos sabores ácido e doce a partir do 6 dia de análise. O odor geral sofreu variação no 7을 dia e os demais parâmetros não sofreram alterações.

Quanto ao iogurte sabor mangaba verificou-se diferença significativa apenas em relação à consistência na boca e aparência no 6o dia.

Os resultados mostraram que para o iogurte sabor pequi houve aumento significativo do sabor ácido a partir do 6o dia e de adstringência no $7^{\circ}$ dia. Observou-se ainda que o odor da fruta diminuiu significativamente no 7음 dia e os demais parâmetros não sofreram alterações.

Os iogurtes sabor buriti e cagaita não apresentaram variações significativas quanto aos atributos analisados.

As alterações sensoriais, da mesma forma que as variações no $\mathrm{pH}$ e acidez titulável já explicadas anteriormente, também podem ser atribuídas ao desenvolvimento de bactérias utilizadas no processamento dos produtos (ALKADAMANY et al., 2002).

\section{CONCLUSÃO}

As análises físico-químicas e sensoriais dos iogurtes com adição de frutos do Cerrado evidenciaram estabilidade dos produtos sob refrigeração por curto período de tempo, que variou de $5 \mathrm{a}$ 6 dias, pois não houve adição de conservantes.

Sobre as análises microbiológicas pode-se concluir que a metodologia e condições de boas práticas de produção adotadas foram adequadas e suficientes para garantir a qualidade microbiológica dos produtos, observando-se que a presença de coliformes totais também pode ter influenciado as variações de $\mathrm{pH}$ e acidez.

Os produtos elaborados constituem boa alternativa alimentar, pois além de alto valor nutritivo, os frutos usados na saborização são muito apreciados pela população da região. Acredita-se que esse fator tenha contribuído para a boa aceitação dos produtos, conforme demonstrado nos testes de aceitabilidade.

Os produtos são viáveis economicamente, pois além de fácil elaboração e baixo custo de produção, grande parte das espécies nativas do Cerrado apresentam elevado potencial para aproveitamento econômico.

\section{ABSTRACT}

\section{ELABORATION AND EVALUATION OF YOGURT WITH CERRADO FRUITS TASTE}

Yogurts with the addition of araticum, buriti, cagaita, jatobá, mangaba and pequi marmalades were processed, verifying their shelf-life, preference and acceptance. Three formulations of the yogurts were elaborated, containing $15 \%, 20 \%$ and $25 \%$ of the marmalades for the yogurts of pequi and jatoba and $20 \%, 25 \%$ and $30 \%$ of the 
marmalades for the other yogurts. The marmalades were added to the natural yogurt in the temperature of $\pm 20^{\circ} \mathrm{C}$ and homogenized. After preparation, the samples were cooled to $4^{\circ} \mathrm{C}$ and submitted to preference analysis. The preferred sample was submitted to acceptance analysis with 100 non trained judges between 8 and 65 years, by hedonic scale of nine points. The shelf life of the yogurts was verified during 7 days by means of sensorial analysis with a team composed of 7 trained judges, physical-chemical analysis for $\mathrm{pH}$ and titratable acidity determination and microbiological analysis for total coliforms, coliforms at $45^{\circ} \mathrm{C}$, Staphylococcus coagulase positive and the presence of Salmonella sp. in $25 \mathrm{~g}$. The results showed good acceptance of the yogurts, the one with araticum taste (with $25 \%$ of marmalade) presented the higher average (7.4). However the yogurt with jatoba taste with $20 \%$ of marmalade revealed inferior average (5.49). The physical-chemical and sensorial analysis demonstrated that the products were stable under refrigeration for a period of 5 to 6 days, characterizing them as products with fast consumption. The microbiological analysis showed that the good manufacturing practices were adequate to guarantee the microbiological quality of the products.

KEY-WORDS: YOGHURT; SENSORIAL ANALYSIS; SHELF-LIFE; CERRADO PRODUCTS.

\section{REFERÊNCIAS}

1 ADOLFSSON, O.; MEYDANI, S.N.; RUSSELL, R.M. Yogurt and gut function. Am. J. Clin. Nutr., v. 80, p.245-56, 2004.

2 ALKADAMANY, A.; TOUFEILI, I.; KHATTAR, M.; ABOU-JAWDEH, Y.; HARAKEH, S.; HADDAD, T. Determination of shelf life of concentrated yogurt (Labneh) produced by in-bag straining of set yogurt using hazard analysis. J. Dairy Science, n. 85, p. 1023-1030, 2002.

3 BRANDÃO, S.C.C. Tecnologia da produção industrial de iogurte. Leite \& Derivados, v.4, n.25, p.24-38, 1995.

4 BRASIL. Ministério da Agricultura Pecuária e Abastecimento. Resolução no 5 de 13/11/00. Padrões de Identidade e Qualidade (PIQ) de Leites Fermentados, Disponível em: <http//www.agricultura.gov.br>. Acesso em: 20/01/2002.

5 BRASIL. Ministério da Saúde. RDC ANVISA/MS $n^{\circ}$. 12, de 02 de janeiro de 2001. Regulamento técnico sobre os padrões microbiológicos para alimentos. Diário Oficial [da] República Federativa do Brasil, Brasília, 10 jan. 2001. Seção 1.

6 CANZI, E.; CASIRAGHI, M.C; ZANCHI, R.; GANDOLFI, R.; FERRARI, A.; BRIGHENTI, F.; BOSIA, R.; CRIPPA, A.; MAESTRI, P.; VESELY, R.; SALVADORI, B.B. Yogurt in the diet of the elderly: a preliminary investigation into its effect on the gut ecosystem and lipid metabolism. Le Lait, v. 82, n. 6, p.713-723, 2002

7 CASTRO, L.T; TEIXEIRA, L.; CALDEIRA, M.; NEVES, M.F.; CONSOLI, M.A. Challenges for increasing milk and yogurt consumption in Brazil. Disponível em: http://www.ifama.org/conferences/2006Conference/SymposiumFinal/ 1091_Paper.pdf. Acesso em: 30/03/2007

8 CHAVES, J.B.P.; SPROESSER, R.L. Práticas de laboratório de análise sensorial de alimentos e bebidas. Viçosa: Imprensa Universitária, 1996.

9 CONSUMO de iogurte. Revista Leite B, v.11, n.123, p.6, 1997.

10 EUCLYDES, R.F. Sistema para análise estatística SAEG. Versão 7.0, Viçosa: UFV, 2000.

11 GODEL, J. Treatment of diarrheal disease. Pediatrics \& Child Health, v. 8, n. 7, p. 455-458, 2003.

12 FARIA, E. V.; YOTSUYANAGI, K. Técnicas de análise sensorial. Campinas: ITAL/LAFISE, 2002.

13 FERNANDÉZ, S.G.; SERRA, M.B.; CARAS, M.A.; BES, J.M.L.; POL, J.R.; GAY, J.R.; GAY, E.T.; PICO, A.R.; LÓPEZ, P.B. Alteraciones reológicas em processos fermentados lácteos. Alimentaria, v. 31, n. 254, p.41-48, 1994.

14 GARCIA, T.B; RUIZ, L.R.A.; DÍAZ, M.E. Microbiologia sanitária de los yogurtes naturales y com sabores de consumo em la província de Alicante. Alimentaria, v. 23, n.177, p. 39-42, 1986.

15 GARCIA-MARTÍNEZ, M; SEARA, P.J.S; GORDILLO-OTERO, M.J; LÓPEZ, M.A.A.; ROJAS, R.M. Valoración nutricional de la composición mineral de yogurtes enteros aromatizados. Alimentaria, v. 35, n. 297, p. 73-76, 1998.

16 GUIA para elaboração do plano APPCC: frutas, hortaliças, e derivados. 2.ed. Brasília: SENAI/DN, 2000. (Série Qualidade e Segurança Alimentar, Projeto APPCC Indústria, Convênio SNI/SEBRAE).

17 IAL. Instituto Adolfo Lutz. Normas analíticas do Instituto Adolfo Lutz: métodos químicos e físicos para análise de alimentos. 3.ed. São Paulo, 1985. v. 1. 
19 JAMES, W.; ANDERSON, M.D; GILLILAND, S.E. Effect of fermented milk (yogurt) containing Lactobacillus acidophilus L1 on Serum Cholesterol in hypercholesterolemic humans. Journal of the American College of Nutrition, v. 18, n.. 1, p. 43-50, 1999.

20 KARAGUL-YUCEER, Y; COGGINS, P.C; WILSON, J.C; WHITE, C.H. Cabonated yogurt - sensory properties and consumer acceptance. J. Dairy Sci., v. 82, p. 1394-1398, 1999.

21 LÓPEZ, M.A.A.; COSANO, G.Z. Valor nutricional de productos lácteos: contenido mineral. Alimentaria, v. 33 n. 265 , p. 37-40, 1995.

22 LÓPEZ, M.C; MEDINA, L.M; CÓRDOBA, M.G; JORDANO, R. Evaluación de la calidad microbiológica en helado de yogur. Alimentaria, v. 35, n. 288, p. 39-45, 1997.

23 LOURENS-HATTINGH, A.; VILJOEN, B.C. Yogurt as probiotic. Int. Dairy Journal, v. 11, n. 1-2, p. 1-17, 2001.

24 MARTINEZ, I.; SANTAELLA, M.; ROS, G.; PERIAGO, M.J. Content and in vitro availability of Fe, Zn, Mg, Ca and P in homogenized fish-based weaning foods after bone addition. Food Chem., v. 63, n. 3, p. 299-305, 1998.

25 MASON, S. Dairy products consumption trends. Western Dairy Digest, v. 7, n. 4, p.3, 2006. Disponível em: www.westerndairyscience.com. Acesso em: 05/01/2007.

26 MENOLI, A.P.V.; FANCHINI, P.T.; DUARTE, D.A.; FERREIRA, S.L.M; IMPARATO, J.C.P. Nutrição e desenvolvimento dentário. Revista de Ciências Biológicas e da Saúde, v. 9, n. 2, p. 33-40, 2003.

27 MOREIRA, S.R; SCHWAN, R.S; CARVALHO, E.P; FERREIRA C. Análise microbiológica e química de iogurtes comercializados em Lavras - M.G. Ciênc. Tec. Alim., v. 19, n. 1, p. 147-152, 1999.

28 NEWELL, G.J; MAC FARLANE, J.D. Expanded tables for multiple comparison procedures in the analysis of ranked data. Journal of Food Science, v. 52, n. 6, p.1721-1722, 1987.

29 ORTIZ, S. A. Aspectos legais da vida-de-prateleira de produtos alimentícios. Boletim do Instituto de Tecnologia de Alimentos, v.19, n. 1, p. 33-87, 1982.

30 PEDRO, N.A.R.; OLIVEIRA, E.; FILLI, S.P.; MONTEIRO, D.M.P. Estudo do conteúdo mineral de iogurtes naturais e com sabor de frutas, comercializados na cidade de São Paulo, Brasil. Archivos Latinoamericanos de Nutricion, v. 51, n. 2, p. 210-215, 2001.

31 PORTO, J.A.; PICCOLI, C.; SALERNO, M.; HENRIQUE, I.T. Raquitismo carencial - relato de caso. Scientia Medica, v. 15, v. 2, p.112-115, 2005.

32 ROCHA, C.; SIQUEIRA, M.I.D; COBUCCI, R.M.A.; SILVA, F.D.; PEIXOTO, K.L; SANTANA, L.V.G. Iogurte de leite de búfala sabor frutos do Cerrado. Boletim do CEPPA, v. 22, n.1, p. 97-106, 2004

33 ROJAS, R.M; RUIZ, C.C; LÓPEZ, M.A; COSANO, G.Z. Contenido mineral del yogurt natural. Alimentaria, v. 30, n. 239, p. 81-84, 1993.

34 SÁNCHEZ-SEGARRA, P.J.; GARCÍA-MARTÍNEZ, M.; GORDILLO-OTERO, M.J.; DÍAZ-VAVERDE, A.; AMARO-LOPEZ, M.A.; MORENO-ROJAS, R. Influence of the addition of fruit on the mineral content of yogurts: nutritional assessment. Food Chemistry, v. 70, p. 85-89, 2000.

35 SAS for Windows. Versão 8. Torrance, CA: Microsoft Coorporation, 1999. 1 CD-ROM

36 SILVA, J.A. Tópicos da tecnologia de alimentos. São Paulo: Varela, 2000.

37 SILVA, N.; JUNQUEIRA, V. C. A.; SILVEIRA, N. F. A. Manual de métodos de análise microbiológica de alimentos - ITAL. São Paulo: Varela, 1997.

38 VANDERZANT, C.; SPLITTSTOESSER, D.F. (ed.). Compendium of methods for the microbiological examination of foods. $3^{\text {rd }}$ ed. Washington (DC): American Public Health Association, 1992.

39 VARNAM, A.H; SUTHERLAND, J.P. Leche y productos lácteos: tecnología, química y microbiologia. Zaragoza (Espana): Editorial Acribia,1995. 476 p.

40 WONG, N.P.; LACROIX, D.E.; ALFORD, J.A. Mineral content of dairy products. J. Am. Diet Assoc., v. 72, n. 3, p. 288291, 1978.

\section{AGRADECIMENTOS}

Os autores agradecem ao CNPq pelo apoio financeiro e bolsas de iniciação científica e à Pró-Reitoria de Pós-Graduação e Pesquisa da Universidade Católica de Goiás pelas bolsas de Iniciação Científica do programa BIC/CP/PROPE. 